\title{
Mass Sensing for the Advanced Fabrication of Nanomechanical Resonators
}

\author{
G. Gruber, ${ }^{\dagger}$ C. Urgell, ${ }^{\dagger}$ A. Tavernarakis, ${ }^{\dagger}$ A. Stavrinadis, ${ }^{\dagger}$ S. Tepsic, ${ }^{\dagger}$ C. Magén, ${ }^{\ddagger}, \S$ S. Sangiao, ${ }^{\ddagger}, \S(0)$ \\ J. M. de Teresa, ${ }^{\ddagger}, 8$ P. Verlot, ${ }^{\|}$and A. Bachtold ${ }^{*}{ }^{\dagger}$ (i)
}

${ }^{\dagger}$ ICFO - Institut de Ciencies Fotoniques, The Barcelona Institute of Science and Technology, 08860 Castelldefels, Barcelona, Spain

${ }^{\ddagger}$ Instituto de Ciencia de Materiales de Aragón (ICMA), Universidad de Zaragoza-CSIC, 50009 Zaragoza, Spain

${ }^{\S}$ Laboratorio de Microscopías Avanzadas (LMA), Instituto de Nanociencia de Aragón (INA), Universidad de Zaragoza, 50018

Zaragoza, Spain

"School of Physics and Astronomy, The University of Nottingham, University Park, Nottingham NG7 2RD, United Kingdom

Supporting Information
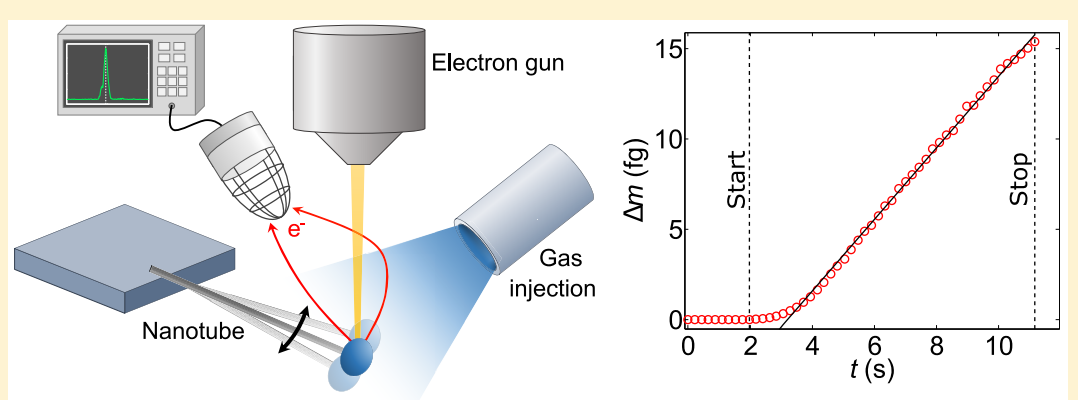

ABSTRACT: We report on a nanomechanical engineering method to monitor matter growth in real time via e-beam electromechanical coupling. This method relies on the exceptional mass sensing capabilities of nanomechanical resonators. Focused electron beam-induced deposition (FEBID) is employed to selectively grow platinum particles at the free end of singly clamped nanotube cantilevers. The electron beam has two functions: it allows both to grow material on the nanotube and to track in real time the deposited mass by probing the noise-driven mechanical resonance of the nanotube. On the one hand, this detection method is highly effective as it can resolve mass deposition with a resolution in the zeptogram range; on the other hand, this method is simple to use and readily available to a wide range of potential users because it can be operated in existing commercial FEBID systems without making any modification. The presented method allows one to engineer hybrid nanomechanical resonators with precisely tailored functionalities. It also appears as a new tool for studying the growth dynamics of ultrathin nanostructures, opening new opportunities for investigating so far out-of-reach physics of FEBID and related methods.

KEYWORDS: Mechanical resonators, NEMS, nanofabrication, mass sensing, carbon nanotube, electron microscopy

$\mathrm{N}$ anomechanical devices are exquisite sensors of mass deposition $^{1-4}$ and external forces. $^{5-9}$ These sensing capabilities enabled advances in mass spectrometry, ${ }^{10-12}$ surface science, ${ }^{13-19}$ scanning probe microscopy, ${ }^{20,21}$ and magnetic resonance imaging. ${ }^{22-24}$ The highest sensitivity is achieved with carbon nanotube resonators ${ }^{4,8}$ because of their tiny mass compared to the other operational mechanical resonators. However, a general challenge with such small transducers is to provide them with a physical function, which can be, for example, magnetic, chemical, or optical. Conventional nanofabrication processes, such as electron-beam lithography and reactive-ion etching, are difficult to employ with such small suspended structures without altering their sensing capabilities. Developing new methods to engineer nanoscale resonators with high precision and providing them with a specific functionality is in high demand as it would enable a whole range of new technological and scientific applications.

In this work, we report a nanofabrication method enabling ultrasensitive, versatile functionalization of carbon nanotube resonators ${ }^{25-27}$ inside a scanning electron microscope (SEM). Using focused electron beam induced deposition (FEBID), ${ }^{28-32}$ we report the mass-controlled growth of $\mathrm{Pt}$ particles on carbon nanotube nanomechanical sensors, enabling their optomechanical funcionalization. ${ }^{33}$ The deposited mass is tracked in real time by monitoring frequency changes of the noise-driven oscillations of the nanotube resonator. Measuring the nanomechanical vibrations relies on

Received: June 10, 2019

Revised: $\quad$ August 7, 2019

Published: September 3, 2019 

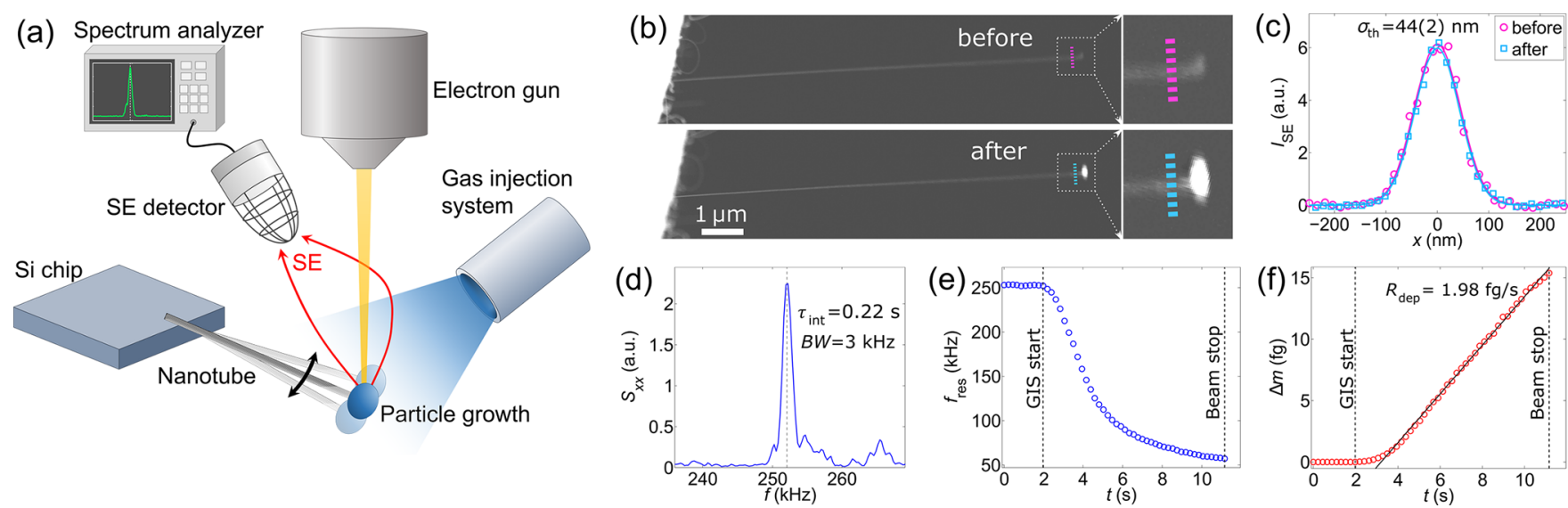

Figure 1. (a) Schematic of the setup. The electron beam is set on the apex of the suspended nanotube cantilever, generating a secondary electron (SE) current, which is detected and fed into a spectrum analyzer. Using the gas injection system (GIS), a nanoparticle is grown on the nanotube, resulting in a shift of the observed resonance frequency. (b) SEM images of a nanotube before and after the deposition of a particle, with $3 \times$ magnified view of the apex (right side). (c) Profiles of the SE current $I_{\mathrm{SE}}$ along the dashed lines marked in (b) with Gaussian fits (solid lines). (d) Typical resonance signal used to count the resonance frequency. (e) Monitoring of the resonance frequency during the deposition; at $t \approx 2 \mathrm{~s}$, the GIS valve was opened, and at $t \approx 11 \mathrm{~s}$ it was closed and the beam exposure stopped. (f) Deposited mass determined from (e) using eq 3.

e-beam electromechanical coupling ${ }^{34,35}$ and is accomplished using the same electron-beam as that used for FEBID. We demonstrate the high sensitivity and versatility of this method, which enables us to address mass changes over more than 6 orders of magnitude with a resolution down to the $\mathrm{zg}$ range.

The samples consist of carbon nanotubes grown via chemical vapor deposition on silicon substrates. The nanotubes stick to the surface due to van der Waals forces. Some nanotubes extend over the substrate edge, forming cantilevers. We used cantilevers with lengths between 1 and $15 \mu \mathrm{m}$ and spring constants between $10^{-7}$ and $2.6 \times 10^{-4} \mathrm{~N} / \mathrm{m}$ in order to investigate the robustness of our method.

All SEM and FEBID experiments were conducted in a Zeiss Auriga field emission electron microscope equipped with a gas injection system (GIS). The acceleration voltage of the electron beam was $5 \mathrm{kV}$ and the typical beam current was $200 \mathrm{pA}$. The precursor gas was methylcyclopentadienyl(trimethyl)platinum(IV) in order to grow a Pt deposit onto the sample surface when illuminated by the electron beam. ${ }^{36}$ All the experiments reported below have been completed with the GIS nozzle being positioned $\approx 500 \mu \mathrm{m}$ away from the substrate.

A schematic of the experimental setup used for the deposition experiments is depicted in Figure 1a. The electron beam is set onto the apex of the nanotube in spot mode while monitoring the secondary electron (SE) current $I_{\mathrm{SE}}$. The signal is displayed in the frequency domain via fast Fourier transform (FFT). The data are real-time processed using a fast peaksearch custom computer program, enabling us to extract the mechanical resonance frequency at a rate typically ranging from 0.5 to $5 \mathrm{~Hz}$.

Figure $1 \mathrm{~b}$ shows a nanotube before and after the deposition process with the deposited particle clearly visible. Furthermore, the free end of the nanotube appears blurred due to the motion fluctuations. The spring constant $k$ can be extracted from the variance of the displacement $\sigma_{\text {th }}^{2}$ using the equipartition theorem

$$
k=\frac{k_{\mathrm{B}} T}{\sigma_{\mathrm{th}}^{2}}
$$

where $k_{\mathrm{B}}$ is the Boltzmann constant and $T$ is the temperature. ${ }^{34}$ Figure 1c shows the SE current profiles taken along the dashed lines marked in Figure $1 \mathrm{~b}$ before and after the deposition with Gaussian fits, which are used to determine $\sigma_{\mathrm{th}}^{2}$. The inferred spring constant $k=2.1(2) \times 10^{-6} \mathrm{~N} / \mathrm{m}$ is the same in both cases. This shows that $k$ is not affected by the deposition process, so that any permanent changes in the mechanical resonance frequency are associated with mass deposition (see further discussion below). Specific care was dedicated to avoid broadening of the observed motion probability density function, which can be induced by back-action phenomena during imaging. ${ }^{34}$ This was achieved by averaging multiple frames using the fastest available scanning speed (122 ms/ frame).

The mass of the Pt particle is monitored in real time during its formation. This is done by continuously acquiring the resonance spectrum of the noise-driven vibrations of the nanotube with the electron beam. We typically use large resolution bandwidth settings in order to enable a high sampling rate. Figure $1 \mathrm{~d}$ shows a typically obtained signal used to count the frequency for the mass detection. The resolution bandwidth of the measurement in this case was $\mathrm{BW}=3 \mathrm{kHz}$. The resonance frequency $f_{\text {res }}$ relates to the effective mass $m^{*}$ of the mechanical eigenmode via the equation

$$
f_{\text {res }}=\frac{1}{2 \pi} \sqrt{\frac{k}{m^{*}}}
$$

Figure 1e shows the evolution of $f_{\text {res }}$ over time. Here, the GIS nozzle was opened at $t \approx 2 \mathrm{~s}$. The electromechanical interaction then becomes strongly nonlinear, resulting in a strong amplification of the electromechanical spectrum and the appearance of a large number of peaks at multiples of the fundamental resonance frequency (not shown here, see Section 1 of Supporting Information). We attribute this behavior to the increasing interaction volume resulting from the deposition process. Our frequency counting algorithm includes a dynamical discrimination procedure enabling one to unambiguously keep track of the fundamental resonance frequency in real time. As shown on Figure 1e, $f_{\text {res }}$ decreases over time, which is the expected evolution in the presence of mass adsorption. 

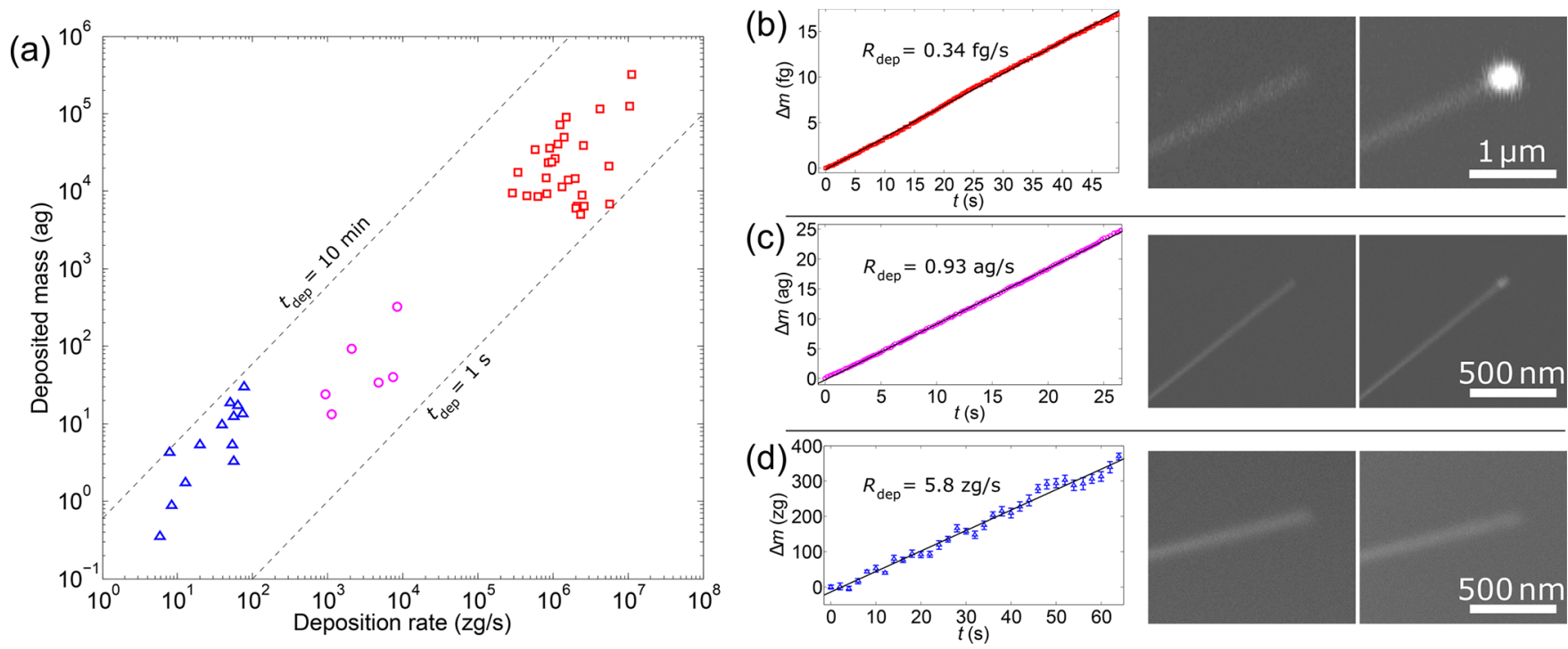

Figure 2. (a) Deposition rate and deposited mass for all the fabricated devices with deposition times $t_{\text {dep }}$ in the range between $1 \mathrm{~s}$ and $10 \mathrm{~min}$. The different operation modes are marked by different colors, and exemplary measurements are shown in (b-d). (b) Mass deposition in default GIS operation mode (GIS nozzle open, precursor in the chamber at a pressure in the range of $p=(7-11) \times 10^{-6} \mathrm{mbar}$ ). (c) Mass deposition in lowpressure mode (GIS nozzle closed, precursor residuals in the chamber with $p=(1-1.7) \times 10^{-6} \mathrm{mbar}$ ). (d) Mass deposition in the background vacuum regime (after more than $24 \mathrm{~h}$ of pumping, $\left.p=(0.8-1) \times 10^{-6} \mathrm{mbar}\right)$. The SEM images on the right show each nanotube before and after the deposition. The spring constants determined before and after the deposition are $k=6.2(5) \times 10^{-7} \mathrm{~N} / \mathrm{m}$ for $(\mathrm{b}), k=1.57(7) \times 10^{-5} \mathrm{~N} / \mathrm{m}$ for (c), and $k=1.00(3) \times 10^{-6} \mathrm{~N} / \mathrm{m}$ for $(\mathrm{d})$.

The deposition was limited to the apex of the nanotube, such that the spring strength can be reasonably assumed to remain unchanged. Therefore, the deposited mass $\Delta m(t)$ yields to a frequency shift, independent from the shape of the eigenmode $e^{10}$

$$
\Delta m(t)=\frac{k}{4 \pi^{2}}\left(\frac{1}{f_{\text {res }, \mathrm{t}}^{2}}-\frac{1}{f_{\text {res }, 0}^{2}}\right)
$$

where $f_{\text {res, },}$ and $f_{\text {res }, 0}$ are the resonance frequencies measured during the deposition at time $t$ and prior to the deposition, respectively. ${ }^{1-4,10-14,16,18}$ In the limit of high signal-to-noise ratio, the mass determination does weakly depend on the SE emission rate. Additionally, we performed optomechanical measurements ${ }^{33}$ in order to gain independent confirmation of the postdeposition mechanical properties (Section 2 of Supporting Information). These measurements ensure that the electromechanical coupling has negligible impact on the mechanical resonance frequency and that the observed changes are due to mass deposition.

Figure If displays the corresponding evolution of the deposited mass over time. After some transient regime, the deposition becomes linear in time, allowing us to extract the deposition rate $R_{\mathrm{dep}}=1.98 \mathrm{fg} / \mathrm{s}$. At $t \approx 11 \mathrm{~s}$, the GIS valve was closed and the beam exposure was stopped to avoid spurious growth. The resonance frequency at the end was $f_{\text {res }}=56.1(5)$ $\mathrm{kHz}$ and the total mass of the particle seen in Figure $1 \mathrm{~b}$ is $(15.5 \pm 2.0) \mathrm{fg}$. Optomechanical measurements of this resonator yield to a postdeposition mechanical resonance frequency $f_{0}=57.04 \mathrm{kHz}$ with a quality factor $Q \approx 3000$ at room temperature (Section 2 of Supporting Information). Besides further confirming the mass-induced origin of the measured frequency change, this measurement demonstrates that the deposition using FEBID does not degrade the mechanical properties of the nanotube resonator, which is crucial in the context of functionalizing nanomechanical resonators.

Using the above-described methodology, a large set of hybrid nanotube cantilevers was fabricated and characterized. Figure $2 \mathrm{a}$ shows the determined deposition rates and final masses of the deposited particles for each experiment. The dashed lines indicate how the deposition rate and deposited mass are related via the deposition duration. The observed variations arise from different modes of operations (see further discussion below) to which have been assigned distinct colors. Note that even within the same mode of operation, the obtained results are widely dispersed. This is because FEBID is a highly complex process where various interdependent parameters may affect the growth rate. ${ }^{37}$ These include the focus of the electron beam, the temperature of the substrate, the temperature and flux of the precursor molecules, and the pressure of residual gas in the chamber. The deposition rate is also affected by the amplitude of the nanotube vibrations, which may be larger than the electron-beam diameter, resulting in a net decrease of the effective deposition cross-section. The different GIS operation modes as well as illustrative results are discussed in the following.

We start with the default operation mode of the GIS, which was also used for the measurements in Figure 1. When the nozzle is opened the precursor gas is released into the chamber resulting in a strong increase of the chamber pressure. The pressure typically saturates in the range $p=(7-11) \times 10^{-6}$ mbar, while the background vacuum pressure is typically $\approx 1 \times$ $10^{-6}$ mbar. It results in measured deposition rates between $0.28 \mathrm{fg} / \mathrm{s}$ and $11 \mathrm{fg} / \mathrm{s}$. Figure $2 \mathrm{~b}$ shows a typical measurement in this operation mode, demonstrating a constant deposition rate $R_{\text {dep }}=0.34 \mathrm{fg} / \mathrm{s}$ over a duration as long as $50 \mathrm{~s}$. The deposited mass is more than 30 times larger than the initially measured mass of the nanotube cantilever.

We explored lower Pt deposition rates by reducing the pressure. This is achieved by first purging the GIS nozzle with 

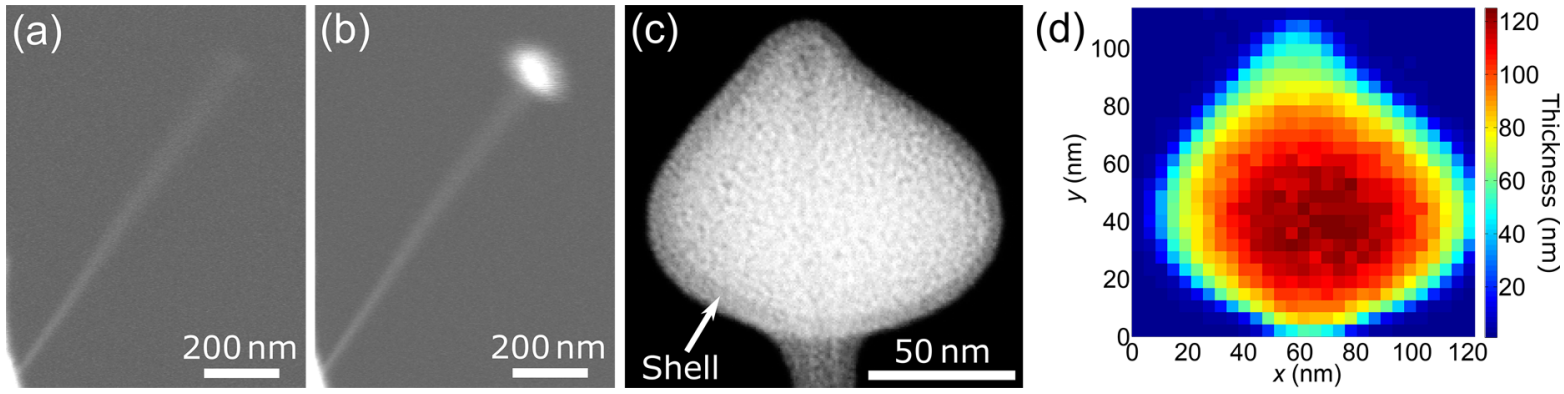

Figure 3. (a,b) SEM images of a nanotube before and after the deposition of a particle with a mass $m_{\text {dep }}=1.33$ fg determined by the resonance frequency measurement. (c) High-angle annular dark-field (HAADF) STEM image of the particle. The visible darker shell is likely the result of the subsequent manipulation of the nanotube with the electron beam (see text). (d) Thickness map of the particle determined by low-loss EELS using the elemental composition of Table 1 and the log-ratio method. ${ }^{38}$

precursor molecules and then pumping the chamber for several minutes. As such, we investigated deposition of precursor molecules in a pressure range $p=(1-1.7) \times 10^{-6} \mathrm{mbar}$ resulting in observed deposition rates ranging between 0.93 $\mathrm{ag} / \mathrm{s}$ and $8.5 \mathrm{ag} / \mathrm{s}$. A typical mass deposition measurement in this low-pressure regime is displayed in Figure 2c. The SEM image after the deposition reveals a small $\mathrm{Pt}$ particle. The deposition rate $R_{\text {dep }}=0.93 \mathrm{ag} / \mathrm{s}$ is equivalent to roughly 2900 $\mathrm{Pt}$ atoms or 1800 precursor molecules per second.

The lowest deposition rates were attained by pumping the chamber for more than $24 \mathrm{~h}$ with the GIS nozzle closed and heated so residual precursor molecules could desorb from the nozzle and be pumped away. It is assumed that in this regime the chamber gas is predominantly composed of organic molecules resulting in e-beam induced deposition of amorphous carbon. The base pressure in this background vacuum regime was in the range $p=(0.8-1) \times 10^{-6} \mathrm{mbar}$ and the observed deposition rates were between 5.8 and $77 \mathrm{zg} / \mathrm{s}$. The lowest value $R_{\text {dep }}=5.8 \mathrm{zg} / \mathrm{s}$ with $2 \mathrm{~s}$ integration time was observed in the experiment shown in Figure $2 \mathrm{~d}$ and is equivalent to about $290 \mathrm{C}$ atoms per second. Computing the Allan deviation of the resonance frequency with $2 \mathrm{~s}$ integration time results in an effective mass resolution of $13 \mathrm{zg}$. This estimation includes the spurious contribution of the deposition of $\mathrm{C}$ atoms, so that it represents an upper bound of the mass resolution of the nanotube resonator. The deposited mass of $330 \mathrm{zg}$ does not result in a distinctive feature on the nanotube in the SEM images (Figure $2 \mathrm{~d}$, right). In this case, the electromechanical measurement enables us to reveal the evolution of the structure that is totally invisible in the SEM image. Besides controlling the growth process, this demonstrates the relevance of e-beam electromechanical coupling as a powerful complementary embedded tool to scanning electron microscopy.

We assessed the material density of a Pt particle and its chemical composition by carrying out scanning transmission electron microscopy (STEM) measurements. The experiments were performed using a $C_{\mathrm{s}}$-corrected FEI Titan transmission electron microscope equipped with a FEI X-FEG high brightness Schottky emitter. The acceleration voltage was 80 $\mathrm{kV}$. Chemical analysis was conducted via energy-dispersive $\mathrm{X}$ ray spectroscopy (EDXS) using an EDAX detector. Thickness measurements were performed by electron energy loss spectroscopy (EELS) employing a Gatan Tridiem 866 ERS energy filter.

A nanotube grown on a STEM copper grid is shown in Figure $3 \mathrm{a}, \mathrm{b}$ before and after depositing a mass of $1.33 \mathrm{fg}$. In a subsequent deposition step, the nanotube was coated with material down to the clamping point to minimize the effects of motion fluctuations. During this step, we avoided exposing the particle directly to the electron beam. Figure $3 c$ displays a highangle annular dark-field (HAADF) image recorded in the STEM and reveals a core-shell structure. The shell appears darker than the core, suggesting that it has a lower density or a lower relative $\mathrm{Pt}$ content than the core particle. It is likely that the shell was formed during the second deposition step and the growth induced by secondary electrons. Previous FEBID works showed that $\mathrm{Pt}$ atoms assemble together to form nanometerscale clusters inside an amorphous $\mathrm{C}$ matrix. ${ }^{36}$

The particle composition was determined by EDXS measurements (Table 1). Platinum accounts for $52.7 \%$ of the

Table 1. Atomic Fraction and Mass Fraction of $\mathrm{C}, \mathrm{Pt}$, and $\mathrm{O}$ Determined by EDXS Measurements of the Particle in Figure 3c

$\begin{array}{lcc}\text { element } & \text { atomic fraction (\%) } & \text { mass fraction (\%) } \\ \text { carbon } & 84.6 & 41.5 \\ \text { oxygen } & 8.8 & 5.8 \\ \text { platinum } & 6.6 & 52.7\end{array}$

mass of the particle. The observed oxygen content is attributed to the air molecules that diffuse into the particle during the transfer of the device from the SEM to the STEM. The atomic $\mathrm{C} / \mathrm{Pt}$ ratio determined by EDXS is $12.8: 1$. This ratio is somewhat larger than the value $8: 1$ reported previously, ${ }^{36}$ suggesting additional amorphous carbon deposition in our experiment, especially during the second deposition step.

Next, we conducted spatially resolved low-loss EELS measurements to map the particle thickness (Figure 3d). The thickness at each point of the map was determined via the log-ratio method using the electron inelastic mean free path determined by the elemental composition in Table $1 .{ }^{38} \mathrm{We}$ obtain the volume $V=5.45 \times 10^{-16} \mathrm{~cm}^{3}$ for the particle core by integrating the thickness over the map surface and by subtracting the volume of the $6 \mathrm{~nm}$ thick shell. This results in the density $\rho \simeq 2.44 \mathrm{~g} / \mathrm{cm}^{3}$ for the particle core using the mass $1.33 \mathrm{fg}$. With this result, we are able to estimate the density of the amorphous C-matrix $\rho_{\mathrm{C}}=\left(\rho-\xi \rho_{\mathrm{Pt}}\right) /(1-\xi)$ where $\xi$ is the normalized atomic $\mathrm{Pt}$ concentration in the pseudobinary composite $\mathrm{Pt}_{\xi} \mathrm{C}_{1-\xi}$ and $\rho_{\mathrm{Pt}}=21.45 \mathrm{~g} / \mathrm{cm}^{3}$ is the bulk density of Pt. ${ }^{39,40}$ Our estimation $\rho_{\mathrm{C}} \simeq 0.95 \mathrm{~g} / \mathrm{cm}^{3}$ compares well with low-quality amorphous (hydro-)carbon deposits, which are typically in the range $(0.3-1.5) \mathrm{g} / \mathrm{cm}^{3} .^{28-30,40,41}$ 
The mass monitoring method opens new possibilities to study the growth of ultrathin nanostructures using FEBID. ${ }^{31,36,40,42-44}$ It may be applied to study how the mass deposition and the material composition depend on experimental growth parameters, such as the electron beam current, the gas-injection rate, and the precursor and substrate temperature. $^{37}$ Our technique is particularly attractive to investigate transients at the beginning of the growth. The good time resolution in the monitoring of the growth rate could be used to test different growth models, for example, involving various precursor dissociation mechanisms (triggered by primary and secondary electrons), precursor coverage, or thermal effects. It may also be employed to test new precursors and to monitor purification steps aiming to improve the material quality. Furthermore, mass monitoring using our method could be applied to study the growth and the milling with a focused ion beam.

In summary, we have reported a method allowing highresolution mass monitoring of the growth of a Pt nanoparticle on a nanotube resonator via in situ electromechanical readout in a FEBID system. The method can be readily employed in any existing SEM or STEM setup without requiring any further modification. The demonstrated mass and time resolution offers a precise control on the deposited mass to engineer nanomechanical sensors, especially since various materials can be grown with FEBID. ${ }^{45,46}$ This may lead to new advances in one- and two-dimensional ${ }^{20,21}$ magnetic force microscopy ${ }^{47}$ and magnetic resonance force microscopy. ${ }^{22,24,48}$ Our technique may also be employed with semiconducting nanowire resonators made from, for example, $\mathrm{GaN}, \mathrm{SiC}$, and InAs ${ }^{35,49-51}$ as well as microfabricated top-down resonators. $^{9,52-58}$

\section{ASSOCIATED CONTENT}

\section{S Supporting Information}

The Supporting Information is available free of charge on the ACS Publications website at DOI: 10.1021/acs.nanolett.9b02351.

Further information regarding nonlinear e-beam electromechanical coupling and independent optomechanical measurement of the postdeposition mechanical resonance frequency (PDF)

\section{AUTHOR INFORMATION}

\section{Corresponding Author}

*E-mail: adrian.bachtold@icfo.eu.

ORCID $\odot$

S. Sangiao: 0000-0002-4123-487X

J. M. de Teresa: 0000-0001-9566-0738

A. Bachtold: 0000-0002-6145-2479

\section{Funding}

This work is supported by the ERC advanced Grant 692876, ERC PoC Grant 862149, the Foundation Cellex, the CERCA Programme, AGAUR, Severo Ochoa (SEV-2015-0522), the Grants FIS2015-69831-P, RTI2018-097953-B-I00, MAT201782970-C2-1-R, and MAT2017-82970-C2-2-R of MINECO, the Fondo Europeo de Desarrollo Regional (FEDER), and the project E13_17R from Aragon Regional Government (Construyendo Europa desde Aragón). P.V. acknowledges support from the ERC starting Grant 758794 "Q-ROOT”.

\section{Notes}

The authors declare no competing financial interest.

\section{ACKNOWLEDGMENTS}

Experimental help by L. Casado from the Laboratory of Advanced Microscopies (LMA) is acknowledged.

\section{REFERENCES}

(1) Yang, Y. T.; Callegari, C.; Feng, X. L.; Ekinci, K. L.; Roukes, M. L. Nano Lett. 2006, 6, 583-586.

(2) Chiu, H.-Y.; Hung, P.; Postma, H. W. C.; Bockrath, M. Nano Lett. 2008, 8, 4342-4346.

(3) Gil-Santos, E.; Ramos, D.; Martinez, J.; Fernandez-Regulez, M.; Garcia, R.; San Paulo, A.; Calleja, M.; Tamayo, J. Nat. Nanotechnol. 2010, 5, 641-645.

(4) Chaste, J.; Eichler, A.; Moser, J.; Ceballos, G.; Rurali, R.; Bachtold, A. Nat. Nanotechnol. 2012, 7, 301-304.

(5) Mamin, H. J.; Rugar, D. Appl. Phys. Lett. 2001, 79, 3358-3360.

(6) Gavartin, E.; Verlot, P.; Kippenberg, T. J. Nat. Nanotechnol. 2012, 7, 509-514.

(7) Moser, J.; Güttinger, J.; Eichler, A.; Esplandiu, M. J.; Liu, D. E.; Dykman, M. I.; Bachtold, A. Nat. Nanotechnol. 2013, 8, 493-496.

(8) de Bonis, S. L.; Urgell, C.; Yang, W.; Samanta, C.; Noury, A.; Vergara-Cruz, J.; Dong, Q.; Jin, Y.; Bachtold, A. Nano Lett. 2018, 18, 5324-5328.

(9) Héritier, M.; Eichler, A.; Pan, Y.; Grob, U.; Shorubalko, I.; Krass, M. D.; Tao, Y.; Degen, C. L. Nano Lett. 2018, 18, 1814-1818.

(10) Hanay, M. S.; Kelber, S.; Naik, A. K.; Chi, D.; Hentz, S.; Bullard, E. C.; Colinet, E.; Duraffourg, L.; Roukes, M. L. Nat. Nanotechnol. 2012, 7, 602-608.

(11) Hanay, M. S.; Kelber, S.; O’Connell, C. D.; Mulvaney, P.; Sader, J. E.; Roukes, M. L. Nat. Nanotechnol. 2015, 10, 339-344.

(12) Dominguez-Medina, S.; Fostner, S.; Defoort, M.; Sansa, M.; Stark, A.-K.; Halim, M. A.; Vernhes, E.; Gely, M.; Jourdan, G.; Alava, T.; Boulanger, P.; Masselon, C.; Hentz, S. Science 2018, 362, 918922.

(13) Wang, Z.; Wei, J.; Morse, P.; Dash, J. G.; Vilches, O. E.; Cobden, D. H. Science 2010, 327, 552-555.

(14) Yang, Y. T.; Callegari, C.; Feng, X. L.; Roukes, M. L. Nano Lett. 2011, 11, 1753-1759.

(15) Atalaya, J.; Isacsson, A.; Dykman, M. I. Phys. Rev. Lett. 2011, 106, 227202.

(16) Tavernarakis, A.; Chaste, J.; Eichler, A.; Ceballos, G.; Gordillo, M. C.; Boronat, J.; Bachtold, A. Phys. Rev. Lett. 2014, 112, 196103.

(17) Rhén, C.; Isacsson, A. Phys. Rev. B: Condens. Matter Mater. Phys. 2016, 93, 125414.

(18) Schwender, J.; Tsioutsios, I.; Tavernarakis, A.; Dong, Q.; Jin, Y.; Staufer, U.; Bachtold, A. Appl. Phys. Lett. 2018, 113, 063104.

(19) Noury, A.; Vergara-Cruz, J.; Morfin, P.; Placais, B.; Gordillo, M. C.; Boronat, J.; Balibar, S.; Bachtold, A. Phys. Rev. Lett. 2019, 122, 165301.

(20) Rossi, N.; Braakman, F. R.; Cadeddu, D.; Vasyukov, D.; Tutuncuoglu, G.; Fontcuberta i Morral, A.; Poggio, M. Nat. Nanotechnol. 2017, 12, 150-155.

(21) de Lépinay, L. M.; Pigeau, B.; Besga, B.; Vincent, P.; Poncharal, P.; Arcizet, O. Nat. Nanotechnol. 2017, 12, 156-162.

(22) Rugar, D.; Budakian, R.; Mamin, H. J.; Chui, B. W. Nature 2004, 430, 329-332.

(23) Degen, C. L.; Poggio, M.; Mamin, H. J.; Rettner, C. T.; Rugar, D. Proc. Natl. Acad. Sci. U. S. A. 2009, 106, 1313-1317.

(24) Nichol, J. M.; Naibert, T. R.; Hemesath, E. R.; Lauhon, L. J.; Budakian, R. Phys. Rev. X 2013, 3, 031016.

(25) Khivrich, I.; Clerk, A. A.; Ilani, S. Nat. Nanotechnol. 2019, 14, $161-167$.

(26) Barnard, A. W.; Zhang, M.; Wiederhecker, G. S.; Lipson, M.; McEuen, P. L. Nature 2019, 566, 89-93. 
(27) Urgell, C.; Yang, W.; de Bonis, S. L.; Samanta, C.; Esplandiu, M. J.; Dong, Q.; Jin, Y.; Bachtold, A. https://arxiv.org/abs/1903. 04892.

(28) Nishio, M.; Sawaya, S.; Akita, S.; Nakayama, Y. J. Vac. Sci. Technol., B: Microelectron. Process. Phenom. 2005, 23, 1975-1979.

(29) Sawaya, S.; Akita, S.; Nakayama, Y. Appl. Phys. Lett. 2006, 89, 193115.

(30) Friedli, V.; Santschi, C.; Michler, J.; Hoffmann, P.; Utke, I. Appl. Phys. Lett. 2007, 90, 053106.

(31) Banerjee, A.; Mankad, T.; Dhamodaran, S.; Ramkumar, J.; Kulkarni, V. N. Nanotechnology 2009, 20, 345501.

(32) Sangiao, S.; Magen, C.; Mofakhami, D.; de Loubens, G.; de Teresa, J. M. Beilstein J. Nanotechnol. 2017, 8, 2106-2115.

(33) Tavernarakis, A.; Stavrinadis, A.; Nowak, A.; Tsioutsios, I.; Bachtold, A.; Verlot, P. Nat. Commun. 2018, 9, 662.

(34) Tsioutsios, I.; Tavernarakis, A.; Osmond, J.; Verlot, P.; Bachtold, A. Nano Lett. 2017, 17, 1748-1755.

(35) Pairis, S.; Donatini, F.; Hocevar, M.; Tumanov, D.; Vaish, N.; Claudon, J.; Poizat, J.-P.; Verlot, P. Phys. Rev. Lett. 2019, 122, 083603.

(36) Wnuk, J. D.; Gorham, J. M.; Rosenberg, S. G.; van Dorp, W. F.; Madey, T. E.; Hagen, C. W.; Fairbrother, D. H. J. Phys. Chem. C 2009, 113, 2487-2496.

(37) Winkler, R.; Lewis, B. B.; Fowlkes, J. D.; Rack, P. D.; Plank, H. ACS Applied Nano Materials 2018, 1, 1014-1027.

(38) Malis, T.; Cheng, S. C.; Egerton, R. F. J. Electron Microsc. Tech. 1988, 8, 193-200.

(39) Utke, I.; Friedli, V.; Michler, J.; Bret, T.; Multone, X.; Hoffmann, P. Appl. Phys. Lett. 2006, 88, 031906.

(40) Friedli, V.; Utke, I.; Mølhave, K.; Michler, J. Nanotechnology 2009, 20, 385304.

(41) Robertson, J. Mater. Sci. Eng., R 2002, 37, 129-281.

(42) Fernandez-Pacheco, A.; De Teresa, J. M.; Cordoba, R.; Ibarra,

M. R. Phys. Rev. B: Condens. Matter Mater. Phys. 2009, 79, 174204.

(43) Pablo-Navarro, J.; Magén, C.; de Teresa, J. M. ACS Applied Nano Materials 2018, 1, 38-46.

(44) Arnold, G.; Winkler, R.; Stermitz, M.; Orthacker, A.; Noh, J.H.; Fowlkes, J. D.; Kothleitner, G.; Huth, M.; Rack, P. D.; Plank, H. Adv. Funct. Mater. 2018, 28, 1707387.

(45) Botman, A.; Mulders, J. J. L.; Hagen, C. W. Nanotechnology 2009, 20, 372001.

(46) Huth, M.; Porrati, F.; Dobrovolskiy, O. V. Microelectron. Eng. 2018, 185-186, 9-28.

(47) Rossi, N.; Gross, B.; Dirnberger, F.; Bougeard, D.; Poggio, M. Nano Lett. 2019, 19, 930-936.

(48) Fischer, R.; McNally, D. P.; Reetz, C.; Assumpcao, G. G. T.; Knief, T.; Lin, Y.; Regal, C. A. New J. Phys. 2019, 21, 043049.

(49) Henry, T.; Kim, K.; Ren, Z.; Yerino, C.; Han, J.; Tang, H. X. Nano Lett. 2007, 7, 3315-3319.

(50) Niguès, A.; Siria, A.; Verlot, P. Nat. Commun. 2015, 6, 8104.

(51) Braakman, F.; Poggio, M. Nanotechnology 2019, 30, 332001.

(52) Yeo, I.; de Assis, P.-L.; Gloppe, A.; Dupont-Ferrier, E.; Verlot, P.; Malik, N. S.; Dupuy, E.; Claudon, J.; Gérard, J.-M.; Auffèves, A.; Nogues, G.; Seidelin, S.; Poizat, J.-P.; Arcizet, O.; Richard, M. Nat. Nanotechnol. 2014, 9, 106-110.

(53) Defoort, M.; Lulla, K. J.; Crozes, T.; Maillet, O.; Bourgeois, O.; Collin, E. Phys. Rev. Lett. 2014, 113, 136101.

(54) Mathew, J. P.; Patel, R.; Borah, A.; Maliakkal, C. B.; Abhilash,

T. S.; Deshmukh, M. M. Nano Lett. 2015, 15, 7621-7626.

(55) Gil-Santos, E.; Baker, C.; Nguyen, D. T.; Hease, W.; Gomez, C.; Lemaître, A.; Ducci, S.; Leo, G.; Favero, I. Nat. Nanotechnol. 2015, 10, 810-816.

(56) Ghadimi, A. H.; Fedorov, S. A.; Engelsen, N. J.; Bereyhi, M. J.; Schilling, R.; Wilson, D. J.; Kippenberg, T. J. Science 2018, 360, 764768.

(57) Roy, S. K.; Sauer, V. T. K.; Westwood-Bachman, J. N.; Venkatasubramanian, A.; Hiebert, W. K. Science 2018, 360, No. eaar5220.
(58) Paulitschke, P.; Keber, F.; Lebedev, A.; Stephan, J.; Lorenz, H.; Hasselmann, S.; Heinrich, D.; Weig, E. M. Nano Lett. 2019, 19, 2207-2214. 